\section{Immunotherapy in Inflammatory Bowel Diseases}

M. Neurath ${ }^{\mathbf{1}}$

${ }^{1}$ Direktor der Medizinischen Klinik 1, Universitätsklinikum Erlangen

Inflammatory bowel diseases are chronic, relapsing, inflammatory disorders of the intestine. Whereas ulcerative colitis is restricted to the colon, Crohn's disease can manifest itself anywhere in the alimentary tract. Ulcerative colitis is characterized by a continuous inflammation of the mucosa and submucosa in the colon. In contrast, Crohn's disease frequently affects all layers of the bowel wall and is associated with segmental, discontinuous inflammation.

Studies in recent years have highlighted a key role of immunotherapy in inflammatory bowel diseases including treatment with immunosuppressive and biological agents. In particular, treatment with the vav1/Rac1 blocker azathioprine is an established immunosuppressive approach for the treatment of inflammatory bowel disease. This treatment is thought to result in $\mathrm{T}$ cell apoptosis with subsequent resolution of gut inflammation in subgroups of patients with inflammatory bowel disease. Furthermore, antibodies against the proinflammatory cytokine TNF have been shown to be effective to induce and maintain remission in patients with these diseases. Adalimumab, infliximab and certolizumab pegol have proven to be particularly effective.

Additional findings in recent years have highlighted the role of additional immune mechanisms in the pathogenesis of inflammatory bowel disease. Among these pathways, the IL-6 pathway appears to play a predominant role by controlling epithelial cell proliferation and augmenting $\mathrm{T}$ cell resistance against apoptosis. It appears that IL-6 perpetuates chronic intestinal inflammation in patients with inflammatory bowel disease. In addition, IL-6 may boost cancer growth in inflammatory bowel disease, as it directly activates the transcription factor STAT3 in intestinal epithelial cells with subsequent cell proliferation.

Several additional immune mechanisms have been identified that appear to contribute to the pathogenesis of inflammatory bowel disease. Among these mechanisms, Th17 cells and their cytokines are potential targets for therapy, although a recent study did not show any benefit of anti-IL-17A therapy in Crohn's disease. Furthermore, IL-21 and IL-23 appear to mediate proinflammatory functions, while IL-22 and IL35 seem to suppress chronic intestinal inflammation.

Based on the above findings, it is likely that we will see additional new anti-inflammatory and immunosuppressive approaches for the therapy of inflammatory bowel disease in the near future.
Conflict of Interest: Lectures and Chairs: Abbott GmbH \& Co. KG/Wiesbaden, Falk Foundation/Freiburg, Ferring Arzneimittel/Kiel, Giuliani Pharma SP./Mailand, A. Pentax Europe $\mathrm{GmbH} /$ Hamburg

Advisor for: Boston Scientific GmbH/Ratingen, Gilead Sciences/Martinsried, GlaxoSmithKline/El Paso, MSD Sharp \& Dohme/Haar, Pentax Europe GmbH/Hamburg, ProtAb Ltd./Israel

\section{References}

1 Perrier C, Rutgeerts P. Cytokine blockade in inflammatory bowel diseases. Immunotherapy 2011; 3: 1341 - 1352

2 Pineton de Chambrun GP, Sandborn WJ. IBD in 2011: advances in IBD management - towards a tailored approach. Nat Rev Gastroenterol Hepatol 2012; 10; 9 (2): $70-72$

3 Neurath MF, Travis SP. Mucosal healing in inflammatory bowel diseases: a systematic review. Gut. 2012 Jul 27 [Epub ahead of print]

4 Neurath MF, Finotto S. IL-6 signaling in autoimmunity, chronic inflammation and inflammation-associated cancer. Cytokine Growth Factor $\operatorname{Rev} 2011 ; 22: 83-89$

\section{Bibliography}

DOI http://dx.doi.org/10.1055/s-0032-1324907

Arzneimittelforschung 2012; 62, Suppl. 1: S13-S13

(c) Georg Thieme Verlag KG Stuttgart · New York .

ISSN 0004-4172 